ISSN 2444-7986

DOI: http://dx.doi.org/10.14201/orl201784.14846

\author{
Artículo de revisión
}

\title{
RESONANCIA MAGNÉTICA DE TIROIDES Y PARATIROIDES
}

\section{Magnetic Resonance Imaging of Thyroid and Parathyroid}

\author{
Miguel GONZALO-DOMíNGUEZ, María Cristina HERNÁNDEZ-RODRíGUEZ, Manuel Ángel \\ MARTIN-PÉREZ, José Martín MARÍN-BALBÍN, Rodrigo BLANCO-HERNÁNDEZ, Ignacio \\ MARTÍN-GARCÍA
}

SACYL: Complejo Asistencial de Zamora. Servicio de Radiodiagnóstico. Zamora. España.

Correspondencia: miguelgonzalodominguez@hotmail.com

Fecha de recepción: 29 de junio de 2016

Fecha de aceptación: 7 de agosto de 2016

Fecha de publicación: 24 de agosto de 2015

Fecha de publicación del fascículo: 1 de diciembre de 2017

Conflicto de intereses: Los autores declaran no tener conflictos de intereses

Imágenes: Los autores declaran haber obtenido las imágenes con el permiso de los pacientes

Política de derechos y autoarchivo: se permite el autoarchivo de la versión post-print (SHERPA/RoMEO)

Licencia CC BY-NC-ND. Licencia Creative Commons Atribución-NoComercial-SinDerivar 4.0 Internacional

Universidad de Salamanca. Su comercialización está sujeta al permiso del editor

RESUMEN

Introducción: El estudio del tiroides y de la paratiroides se realiza habitualmente con ultrasonidos, existiendo sistemas de clasificación internacionalmente aceptados en caso de la patología neoplásica como el sistema TIRADS, y patrones bien definidos de la imagen ecográfica en caso de patología inflamatoria. No obstante, existen ciertas circunstancias en las que está indicado realizar su exploración con tomografía computada o bien con resonancia magnética. Material y Métodos: Realizamos una revisión de las principales indicaciones para la valoración de estas glándulas con resonancia magnética a partir de nuestra experiencia, con los resultados obtenidos en 64 pacientes. Resultados: Establecemos cuáles son los protocolos específicos y las secuencias más adecuadas para su correcta caracterización y planteamos, además, la semiología de los resultados obtenidos mediante esta técnica, haciendo correlación con los procesos patológicos que afectan a estas estructuras cervicales.

PALABRAS CLAVE tiroides; paratiroides; resonancia magnética; protocolos; semiología; secuencias de resonancia magnética.

SUMMARY

Introduction: The assessment of the thyroid and parathyroid pathology is usually achieved with ultrasounds. There are several systems of classification that are internationally accepted in neoplastic disease, such as TIRADS system, and there are well-defined patterns for ultrasound imaging in inflammatory disease. Material and methods: However, there are specific needs that require magnetic resonance imaging. We review the main indications of MRI in the evaluation of thyroid and parathyroid in 64 patients and determine which protocols are more appropriate and which sequences are better for a proper characterization. Results: Then we review the semiology obtained by this technique, making correlation with disease processes affecting these cervical structures.

KEYWORDS

Thyroid; parathyroid; MRI; protocols; semiotics; MRI sequences 


\section{INTRODUCCIÓN}

El estudio de las glándulas tiroides y paratiroides mediante el empleo de resonancia magnética $(\mathrm{RM})$ es poco habitual, ya que la ecografía aporta la información suficiente para establecer el diagnóstico, pero no por ello carece de indicaciones clínicas. Se realiza principalmente en aquellos casos en que la ecografía en primer lugar y la tomografía computada multidetector (TCMD) en segundo lugar han resultados insuficientes para la identificar ciertos procesos patológicos [1, 2].

La ecografía supera ampliamente a las otras técnicas de imagen en la caracterización morfológica de las lesiones tiroideas. El papel fundamental de los ultrasonidos reside en su utilidad para la caracterización morfológica de los nódulos tiroideos, permitiendo valorar el aspecto sólido o quístico de las lesiones, la presencia de calcificaciones y el tipo de vascularización, por lo que se convierte en una herramienta fundamental para establecer una selección adecuada de los nódulos subsidiarios de punción. Algunos autores han planteado un sistema estandarizado de informe de los nódulos tiroideos aceptado ya por varias asociaciones científicas, con el fin de poder estratificar el riesgo de malignidad, basándose en el sistema de clasificación de informes de radiología mamaria (BI-RADS). Este sistema define una serie de criterios y de hallazgos radiológicos recogidos en la clasificación TI-RADS, pero que, a diferencia de la patología de la mama, se encuentra circunscrita exclusivamente a los hallazgos de los estudios ecográficos [3-5].

No obstante, la RM puede presentar una serie de ventajas que se basan, en primer lugar, en una visión ampliada y conjunta de la región cervical y en segundo lugar, en presentar una mayor definición tisular y relación con las estructuras adyacentes. Además, no requieren de la utilización de contrastes yodados y no radian al paciente.

A pesar de ello la RM mantiene un papel secundario en la evaluación de la enfermedad tiroidea, resultando algo más destacable en la valoración de las paratiroides, donde la ecografía presenta más dificultades [6-8].

Los estudios de RM suelen estar especialmente indicados en la estadificación del cáncer de tiroides y de paratiroides, permitiendo valorar mejor la extensión loco-regional de los tumores glandulares, con especial atención en estructuras clave como la tráquea y el esófago, también en la comprobación de signos compresivos y en la evaluación de la posible afectación de las cadenas ganglionares cervicales [9].

Además, permite estudiar la propagación de ciertas patologías a los espacios parafaríngeos, mediastínicos, retrotraqueal e incluso al parénquima pulmonar [10].

La RM también se recomienda para la localización de metástasis ocultas en pacientes post-tiroidectomía, principalmente en el seguimiento de aquellos casos con nivel de tiroglobulina sérica elevada y en el rastreo de lesiones definidas por estudios gammagráficos no visibles por ecografía. Además, es la técnica que mejor nos caracteriza las alteraciones del desarrollo como los quistes del conducto tirogloso o la localización ectópica de las glándulas.

Cabe destacar también el empleo de la tomografía por emisión de positrones (PET) asociada a la TC o RM, que también comienzan a jugar un papel prometedor en la evaluación del cáncer de tiroides y paratiroides.

Teniendo en cuenta estas consideraciones, planteamos realizar una revisión completa de los aspectos fundamentales de la imagen de RM en el estudio de estas glándulas de la región cervical anterior, valorando la anatomía normal, la semiología de la $\mathrm{RM}$ en los procesos patológicos más frecuentes, las principales indicaciones y los criterios de inclusión, teniendo siempre en cuenta que se trata de un recurso limitado en la mayoría de los centros hospitalarios y que supone un coste económico elevado con respecto a otro tipo de pruebas.

\section{MATERIAL Y MÉTODO}

Revisión sobre la aplicación de la RM en el diagnóstico y seguimiento de la patología de tiroides y paratiroides. Se recopiló información acerca de 64 pacientes, con edades comprendidas entre los 40 y 90 años, 50 mujeres y 14 hombres, en el periodo comprendido entre marzo de 2013 y marzo de 2016, a quienes se les solicitó estudio de RM de la región cervical por cuatro motivos principales: sospecha de neoplasia de glándulas tiroides y paratiroides, solicitando establecer dependencia tisular y definir los límites de las lesiones; neoplasias ya confirmadas, para valorar la afectación otras estructuras cervicales o torácicas; alteraciones del desarrollo, como 
las ectopias glandulares y los quistes residuales, y ante bocios voluminosos en los que se querían comprobar posibles signos compresivos. La mayoría de los pacientes contaban con estudios previos, bien de ecografía o de ecografía y TCMD.

\section{PROTOCOLO DE OBTENCIÓN DE IMÁGENES}

Las exploraciones de RM se llevaron a cabo en un equipo Signa Excite de 1.5 Teslas de General Electric mediante la utilización de una antena de cuadratura específica.

El protocolo utilizado se fundamenta en la inclusión de un rango amplio de estudio que se extiende desde la base de cráneo hasta la bifurcación de la tráquea con cortes multiplanares y lo más finos posibles.

El paciente colocado en decúbito supino con la cabeza centrada en línea media y los brazos hacia abajo. Vía venosa en el antebrazo derecho y contraste basado en quelatos de gadolinio (Gd) con una concentración de 0,1 $\mathrm{mmol} / \mathrm{kg}$ a un flujo de $2 \mathrm{ml} / \mathrm{seg}$.

Se realizó en primer lugar una exploración morfológica con secuencias estándar para la región cervical añadiendo una valoración complementaria tras administración de contraste intravenoso.

Nos basamos en secuencias axiales y coronales T1-TSE y T2-TSE, rastreo coronal en secuencias STIR y estudio contrastado con secuencias T1-TEG con saturación grasa.

\section{REVISIÓN DE LA BIBLIOGRAFÍA}

Con el fin de obtener los objetivos fijados, realizamos una revisión los protocolos y técnicas recomendados por las principales sociedades científicas orientados al estudio de las glándulas tiroides y paratiroides, principalmente para la correlación con las aplicaciones disponibles en nuestro centro, para poder evaluar las diferentes pruebas diagnósticas, las indicaciones y los criterios de inclusión [11-13].

\section{CARACTERÍSTICAS ANATÓMICAS DEL} TIROIDES Y PARATIROIDES EN RM

La glándula tiroides presenta dos lóbulos laterales de unos 4 centímetros de longitud cada uno, habitualmente asimétricos, que se unen por una región central de menor volumen llamada istmo, con una pequeña prolongación superior denominada lóbulo piramidal. La glándula tiroidea se localiza entre las capas de la porción media de la fascia cervical profunda, que le sirve de cobertura, además de presentar una cápsula interna propia, muy delgada y adherida al parénquima, que la divide en sus diferentes lóbulos y lobulillos.

Tanto la glándula tiroides como la paratiroides presentan en condiciones normales el aspecto habitual de las estructuras de partes blandas, con parénquima homogéneamente isointenso con respecto al del músculo en las secuencias $\mathrm{T} 1$, indistinguible en las secuencias sin contraste e isointenso o levemente hiperintenso en las secuencias con potenciación T2 y con mínimo realce tras administración de gadolinio.

Las glándulas paratiroides presentan forma de lenteja, habitualmente de situación posterior a la tiroides, aunque su localización puede verse alterada con frecuencia, siendo posible encontrarlas en cualquier punto del eje definido por el ángulo mandibular y la parte inferior del mediastino superior.

Las medidas promedio en condiciones normales son de $6 \mathrm{~mm}$ de longitud, 3 a $4 \mathrm{~mm}$ de diámetro transverso y 1 a $2 \mathrm{~mm}$ de diámetro anteroposterior.

Habitualmente hay cuatro glándulas paratiroides, no obstante, podemos encontrarnos glándulas supernumerarias.

Al igual que el tiroides se encuentran incluidas en la capa media de la fascia cervical profunda.

\section{SEMIOLOGÍA Y ASPECTO DE RM EN LA PATOLOGÍA DE TIROIDES Y PARATIROIDES}

\section{PATOLOGÍA DE TIROIDES}

\section{ADENOMAS Y BOCIO MULTINODULAR}

El uso de la RM en el bocio mutinodular resulta muy limitado, únicamente nos resulta útil en la valoración de la extensión mediastínica y en la definición de los signos compresivos (Figura 1).

El aspecto del bocio en RM es el de una glándula agrandada, heterogénea y multinodular. Alterna áreas de baja intensidad de señal con áreas quísticas y calcificaciones.

Puede presentar nódulos hipercaptantes tras administración de contraste y en caso de bocios de larga evolución es posible encontrar signos de infiltración grasa, con regiones hiperintensas tanto en secuencias potenciadas en T1 como en T2, que representan infiltración lipomatosa tiroidea. Es importante 
establecer diagnóstico diferencial con otras entidades de estirpe grasa en el interior del tiroides como el adenolipoma, que habitualmente se presenta como una lesión encapsulada.

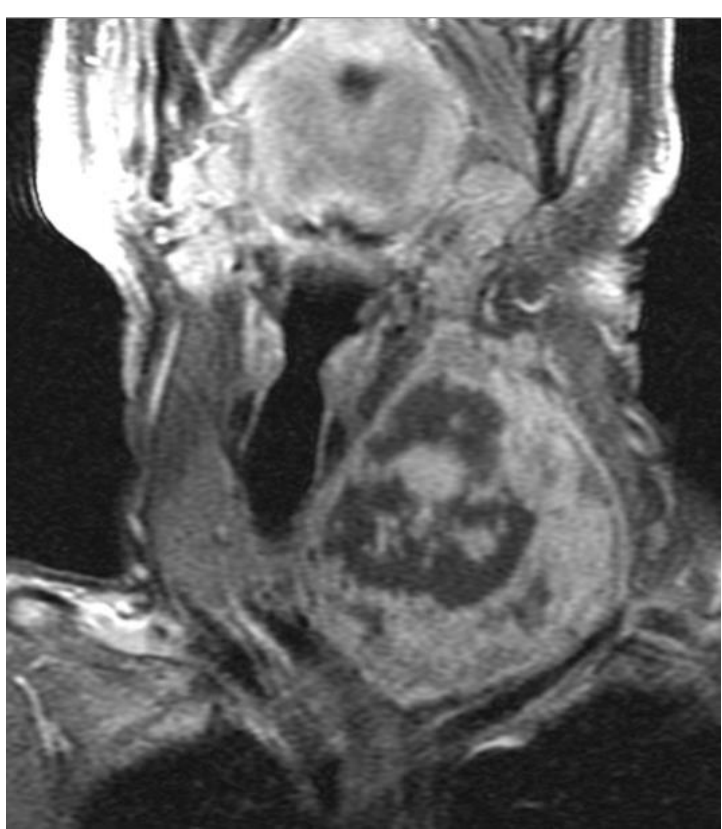

Figura 1. RM en tiroides y paratiroides. Efecto compresivo de un voluminoso bocio tiroideo con compresión y desplazamiento traqueal.

\section{QUISTES TIROIDEOS}

Suelen ser hallazgos casuales en estudios de $\mathrm{RM}$ cervicales realizados con otros fines diagnósticos.

Provienen de la degeneración completa de nódulos hiperplásicos. Son hiperintensos en secuencias potenciadas en T2, con aspecto más variable en las secuencias con potenciación T1, ya que su contenido puede ser coloide, seroso o hemorrágico.

La clave diagnóstica se encuentra en descartar modularidad intraquística o engrosamientos irregulares.

\section{NEOPLASIAS TIROIDEAS}

Las neoplasias primarias del tiroides son el carcinoma papilar, el carcinoma folicular, el carcinoma medular y el anaplásico.

Para la caracterización del nódulo tiroideo la $\mathrm{RM}$ resulta útil en la valoración de los siguientes parámetros:

- $\quad$ Extensión extraglandular (Figura 2).

- Infiltración de órganos vecinos.
- Rastreo ganglionar.

- Estadificación.

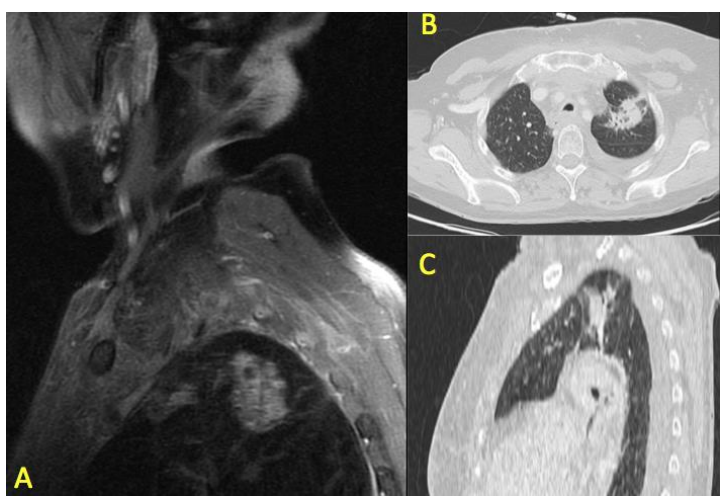

Figura 2. RM en tiroides y paratiroides. Extensión extraglandular. A) Detección de metástasis pulmonares mediante RM cervical en carcinoma medular de tiroides en secuencias Sagital T1. B y C) Confirmación de las metástasis mediante TC torácico.

En el rastreo ganglionar, las neoplasias tiroideas suelen extenderse principalmente a las cadenas supraclaviculares y cadenas yugular baja y media, correspondientes con los niveles III y IV, y la RM complementa de forma muy significativa los rastreos de metástasis que se realizan con técnicas de medicina nuclear, como la gammagrafía y principalmente el PET (Figura 3).

Para la estadificación, la RM presenta una eficacia del 86 al 96\%, con mejor rendimiento en el carcinoma papilar anaplásico y en el medular, que presentan por lo general múltiples microcalcificaciones (cuerpos de psamoma), necrosis y hemorragias. Se reduce en el carcinoma folicular, que resulta más homogéneo, aportando únicamente criterios de tamaño en este caso.

Además, hemos comprobado como la RM aporta información complementaria adicional en las complicaciones post-tratamiento, con la caracterización de áreas de fibrosis, restos tumorales o abscesos (Figura 4).

\section{CARCINOMA PAPILAR}

El más frecuente, supone el $75 \%$ de las neoplasias tiroideas. Habitualmente en mujeres menores de 40 años. Tiene una supervivencia del $90 \%$ a los 20 años y la presencia de adenopatías metastásicas en el momento del diagnóstico no afecta al buen pronóstico. En RM es habitual definir carcinomas multifocales. Se identifican lesiones completamente 
sólidas pudiendo encontrarse degeneración quística en el caso de las adenopatías metastásicas.
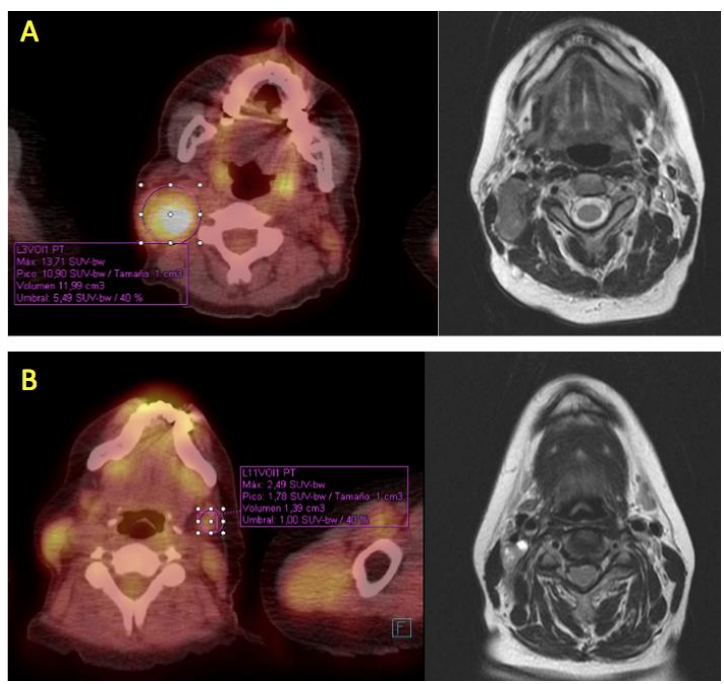

Figura 3. RM en tiroides y paratiroides. Correlación de extensión glandular metástasica de PET con imágenes T1 axial de RM.

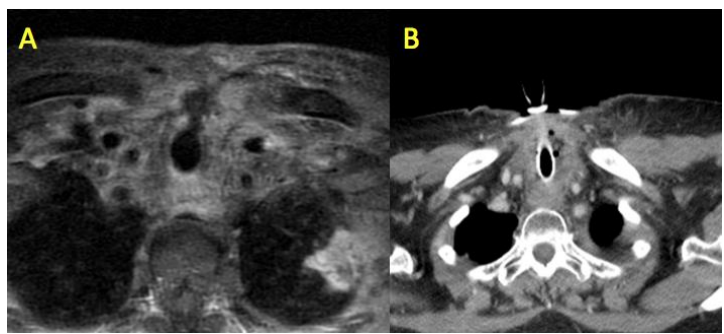

Figura 4. RM en tiroides y paratiroides. Absceso en lecho quirúrgico tras tiroidectomía. A) Imagen por RM con gadolinio. B) Imagen obtenida con TC con contraste.

\section{CARCINOMA FOLICULAR}

Un $10 \%$ de las neoplasias tiroideas. Más frecuente en mujeres a partir de la sexta década de la vida. Buen pronóstico. Suele coexistir con bocio multinodular. En RM indistinguible de los adenomas o nódulos benignos, por lo que la RM no resulta útil para su diagnóstico.

\section{CARCINOMA MEDULAR}

Supone en $5 \%$ de los cánceres de tiroides. Más frecuentes en edades avanzadas. Entre el 10 y $50 \%$ de supervivencia a los 5 años. Hasta un 15\% está incluido en el síndrome de neoplasia múltiple (MEN).

\section{CARCINOMA ANAPLÁSICO}

En RM suele presentarse con infiltración de estructuras vecinas desde el diagnóstico, presentándose como una gran masa heterogénea invasiva y con frecuentes áreas de necrosis intralesionales.

\section{LINFOMA TIROIDEO}

Se presenta como una masa homogénea, sin necrosis ni calcificaciones, con mínimo realce tras administración de contraste.

\section{MetÁstasis}

Las metástasis intraglandulares son muy infrecuentes. Habitualmente se comportan como lesiones encapsuladas. Las entidades que más frecuentemente pueden metastatizar al tiroides son las neoplasias renales, neoplasias pulmonares y de mama.

PATOLOGÍA INFLAMATORIA

En este apartado se incluyen tanto las de origen infeccioso como las autoinmunes.

\section{ENFERMEDAD DE HASHIMOTO}

Se caracteriza por presentar un incremento difuso de la intensidad de señal en secuencias con potenciación T2 y STIR, observándose bandas lineales hipointensas que se extienden por el parénquima en forma de trabéculas, representando áreas de fibrosis.

\section{TIROIDITIS DE RIEDEL}

Es un proceso inflamatorio que se caracteriza por un marcado desarrollo de fibrosis glandular desde el inicio de la enfermedad, que progresivamente puede ir extendiéndose a los tejidos adyacentes. En RM predomina una intensidad de señal muy baja en todas las secuencias, con la práctica ausencia de captación de contraste.

TIROIDITIS GRANULOMATOSO O DE QUERVAIN

Tiroiditis infecciosa. Se caracteriza en RM por una afectación difusa, con incremento de a intensidad de señal difusa en secuencias con potenciación T2 y en STIR, con realce heterogéneo tras administración de contraste. En algunos casos puede presentarse como una lesión focal.

\section{ALTERACIONES DEL DESARROLLO}

\section{QUISTES DEL CONDUCTO TIROGLOSO}

Se producen por fallo en la involución de una parte del conducto por el que desciende la glándula en el desarrollo embrionario. 
Pueden encontrarse a cualquier nivel del trayecto que discurre desde el agujero ciego en la base de la lengua hasta la porción anterior a los lóbulos tiroideos, aunque la situación más frecuente es suprahiodea. Para su localización y la valoración completa del conducto tirogloso es recomendable la utilización se secuencias T1 y T2 en incidencias sagitales.

Se caracterizan por aspecto fundamentalmente quístico, muy intenso en secuencias con potenciación T2 y marcadamente hipointensas en secuencias con potenciación T1, aunque puede resultar variable según la concentración de proteínas que contengan, pudiendo observarse incluso levemente hiperintenso.

Tras administración de contraste pueden presentar leve realce periférico.

\section{GLÁNDULA TIROIDEA ECTÓPICA}

Se produce por descenso incompleto del tiroides hacia la parte inferior del cuello.

Glándula tiroidea habitualmente de aspecto normal, aunque es frecuente que desarrolle quiste coloide. La localización más frecuente es profunda al agujero ciego, en la base de la lengua, tiroides lingual.

\section{PATOLOGÍA DE LAS GLÁNDULAS PARATIROIDES}

Básicamente nos vamos a encontrar adenomas y en menor medida hiperplasia de paratiroides, los carcinomas paratiroideos son excepcionales.

\section{ADENOMAS PARATIROIDEOS}

Principal causa de hiperparatiroidismo primario, mucho más frecuente que la hiperplasia.

Los adenomas se identifican cuando encontramos una glándula paratiroides marcadamente aumentada de tamaño. Se caracterizan por ser hipointensos de forma homogénea en secuencias con potenciación $\mathrm{T} 1$, hiperintensos en secuencias potenciadas en T2 (Figura 5).

\section{HIPERPLASIA DE PARATIROIDES}

De aspecto similar al descrito en los adenomas, aunque afectando de forma general a la mayor parte de las glándulas paratiroides.

\section{CARCINOMA DE PARATIROIDES}

Tumor muy infrecuente. Habitualmente productor de hormonas.

Se establece sospecha de carcinoma de paratiroides, ante una lesión glandular aislada, heterogénea, mal definida y de bordes imprecisos, que incluso puede presentar signos de infiltración de estructuras vecinas.

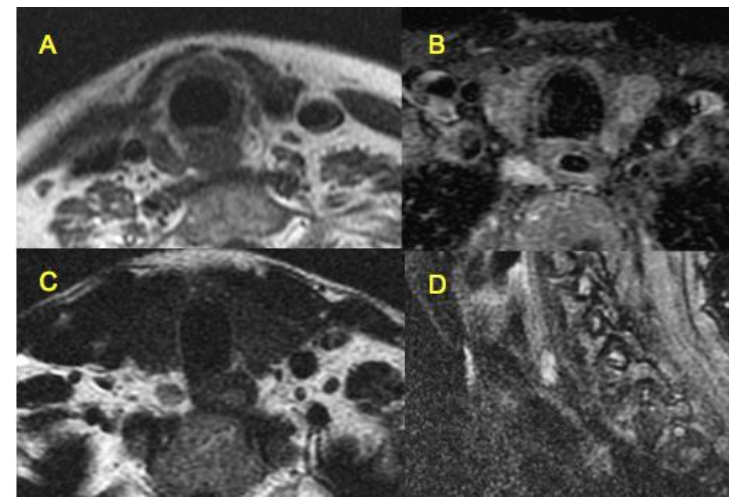

Figura 5. RM en tiroides y paratiroides. Adenoma de paratiroides. A) Secuencia axial T1. B) Secuencia axial STIR. C) Secuencia axial T2. D) Secuencia sagital T1 con contraste.

\section{DISCUSIÓN}

La prueba diagnóstica inicial en el estudio de las glándulas tiroides y paratiroides debe ser siempre la ecografía.

La RM puede aportarnos información complementaria para definir habitualmente hallazgos que pueden resultar incompletos con la aplicación de los ultrasonidos.

Existen cuatro escenarios comunes en las que puede surgir la necesidad de realizar una RM del carcinoma de tiroides: en la detección del nódulo tiroideo incidental, en la evaluación de las metástasis tiroideas nodales, para la obtención de imágenes prequirúrgicas para la enfermedad invasiva y en el seguimiento de recurrencia en el cuello después del tratamiento [14-16].

En muchos casos este tipo de hallazgos radiológicos pudieran ser también obtenidos mediante TCMD, pero el uso de contrastes yodados podría estar contraindicado en pacientes con hipertiroidismo manifiesto, insuficiencia renal, reacciones alérgicas, y sobre todo cuando el paciente vaya a ser sometido a tratamientos con yodo radiactivo.

Los pacientes que van a ser sometidos a un tratamiento con 1131 no deben haber recibido contraste yodado desde al menos 2 meses antes del tratamiento [17].

La RM puede ser prueba morfológica de localización tras la gammagrafía en el rastreo de adenomas de paratiroides y en el rastreo de alteraciones de desarrollo. 
La resonancia magnética nos provee de unas características morfológicas complementarias, en la mayoría de los casos, a los hallazgos estudiados mediante otras técnicas. Sin embargo, observamos que resulta escasamente específica en la patología de más relevancia en los órganos estudiados, el nódulo tiroideo. Consideramos que la RM tiene potencial para una mejor caracterización de dichas lesiones que probablemente vengan de la mano de los estudios de RM avanzada que utilizan protocolos de valoración funcional. Las imágenes de difusión (DWI) pueden ser útiles para diferenciar los nódulos benignos y malignos [18-20].

En recientes estudios observamos que los nódulos benignos tienen mayor coeficiente de difusión aparente (ADC), que los valores de los malignos. La espectroscopia de RM utilizando tiempo de eco (TE) largo ha demostrado ser un método sensible en la diferenciación del carcinoma de tiroides folicular. La presencia de picos de colina es posible identificarla en casi todos los carcinomas, además de la valoración de la proporción de colina/creatina, que va desde valores de 1,6 en los carcinomas diferenciados, a valores de 9,4 en los carcinomas anaplásicos.

El tejido tiroideo normal y lesiones foliculares benignas generalmente no demuestran ningún pico de colina [21-23].

\section{CONCLUSIONES}

La exploración tiroidea ha mejorado desde la introducción de nuevas técnicas ecográficas, gammagráficas, TC, RM y el desarrollo de fusión de imágenes.

La ecografía es la técnica principal para la valoración inicial y para la clasificación de los nódulos tiroideos con el fin de realizar punciones diagnósticas.

La RM es probablemente la técnica más indicada en la estadificación de tumores, en la valoración de signos compresivos u obstructivos y en la localización de alteraciones del desarrollo.

\section{BIBLIOGRAFÍA}

1. Hopkins CR, Reading CC. Thyroid and parathyroid imaging. Semin Ultrasound CT MR 1995;16:279-95.
2. Weber AL, Randolph G, Aksoy FG.The thyroid and parathyroid glands. CT and MR imaging and correlation with pathology and clinical findings. Radiol Clin North Am. 2000;38:1105-29.

3. Wei X, Li Y, Zhang S, Gao M. Thyroid imaging reporting and data system (TIRADS) in the diagnostic value of thyroid nodules: a systematic review. Tumour Biol. 2014; 35(7):6769-76.

4. Ko SY, Lee HS, Kim EK, Kwak JY. Application of the Thyroid Imaging Reporting and Data System in thyroid ultrasonography interpretation by less experienced physicians. Ultrasonography. 2014;33(1):49-57.

5. Montaño-Ascencio PG, García-Baeza LG, Gómez-Vargas E, PérezHernández JU. Valor predictivo positivo del ultrasonido en la clasificación TIRADS. Anales de Radiología México 2014;13:361-8.

6. Hoang JK, Branstetter BF, Gafton AR, Lee WK, Glastonbury CM. Imaging of thyroid carcinoma with $\mathrm{CT}$ and MRI: approaches to common scenarios. Cancer Imaging. 2013;13(1):128-39.

7. Ozkaya M, Elboga U, Sahin E. Evaluation of conventional imaging techniques on preoperative localization in primary hyperparathyroidism . Bosnian Journal of Basic Medical Sciences. 2015;15(1):61-6.

8. Shah S, Win Z, Al-Nahhas A. Multimodality imaging of the parathyroid glands in primary hyperparathyroidism. Minerva Endocrinol. 2008;33(3):193-202.

9. King, AD, Ahuja AT, To EW, Tse GM, Metreweli C. Staging papillary carcinoma of the thyroid: magnetic resonance imaging vs ultrasound of the neck. Clinical radiology. 2000;55(3):222-6.

10. Ersoy R, Karako A, Atasever T. Imaging techniques for metastatic thyroid medullary cancer. Turk $\mathrm{J}$ Endocrinol Metab. 2002;4:149-53.

11. Stark DD, Clark OH, Moss AA. Magnetic resonance imaging of the thyroid, thymus, and parathyroid glands. Surgery. 1984;96(6): 1083-91. 
12. Reading CC, Gorman CA. Thyroid imaging techniques. Clin Lab Med. 1993;13:711-24.

13. Chaudhary V, Bano S. Imaging of the thyroid: Recent advances. Indian Journal of Endocrinology and Metabolism. 2012;16(3):371-6.

14. Kabala JE. Computed tomography and magnetic resonance imaging in diseases of the thyroid and parathyroid. Eur $\mathrm{J}$ Radiol. 2008;66(3):480-92.

15. Gotway MB, Higgins CB. MR imaging of the thyroid and parathyroid glands. Magn Reson Imaging Clin N Am. 2000;8(1):163-82.

16. Soto GD, Halperin I, Squarcia $M$, Lomena F, Domingo MP. Update in thyroid imaging: The expanding world of thyroid imaging and its translation to clinical practice. Hormones (Athens) 2010;9:287-98.

17. Van der Molen AJ, Thomsen HS, Morcos SK. Effect of iodinated contrast media on thyroid function in adults. Eur Radiol 2004;14:902-7.

18. Bozgeyik Z, Coskun S, Dagli AF, Ozkan Y, Sahpaz F, Ogur E. Diffusionweighted MR imaging of thyroid nodules. Neuroradiology 2009;51:193-8.
19. Lu Y, Moreira AL, Hatzoglou V. Using Diffusion-Weighted MRI to Predict Aggressive Histological Features in Papillary Thyroid Carcinoma: A Novel Tool for Pre-Operative Risk Stratification in Thyroid Cancer. Thyroid. 2015;25(6):672-80.

20. Khizer AT, Raza S, Slehria AU. Diffusion-weighted MR Imaging and $A D C$ Mapping in Differentiating Benign from Malignant Thyroid Nodules. J Coll Physicians Surg Pak. 2015;25(11):785-8.

21. Bozgeyik Z, Coskun S, Dagli AF, Ozkan Y, Sahpaz F, Ogur E. Diffusionweighted MR imaging of thyroid nodules. Neuroradiology. 2009;51:193-8.

22. King AD, Yeung DK, Ahuja AT, Tse $\mathrm{GM}$, Chan $\mathrm{AB}$, Lam SS, et al. In vivo MR spectroscopy of thyroid carcinoma. Eur J Radiol 2005;54:112-7.

23. Jordan KW, Adkins CB, Cheng LL, Faquin WC, Application of MagneticResonance-Spectroscopy- Based Metabolomics to the Fine-Needle Aspiration Diagnosis of Papillary Thyroid Carcinoma. Acta Cytologica 2011;55:584-9. 